\title{
Network Issues in the Growth and Adoption of Networked CSCW Services
}

\author{
Roel Vertegaal and Steve Guest
}

\section{Introduction}

Computer Supported Cooperative Work (CSCW) environments have traditionally made heavy use of network technology to allow users (often at different locations) to work together via computer systems. However, this dependency of CSCW applications on the underlying network technology has up to now not been a real issue within the community. CSCW research has traditionally focused on the design of shared environment applications that run on this underlying network, e.g., the development of interaction and presentation techniques for shared tasks. Also, CSCW has been a testbed for design methodologies such as the ethnographic analysis of user behaviour. Although we supporr this emphasis on social science within the field, we feel that in this truly multidisciplinary area researchers should become more aware of networkrelated research issues. A number of parallel events can be identified that triggered our concern:

- The move from Local Area Nerwork technology to Wide Area Network technology (the global Internet) for CSCW applications.

- The evolution of this Internet towards commercialization of services and the privatisation of many telecommunication service providers in Europe.

- The political debate over the Information Super Highway, necessary to support the increasing bandwidth requirements typical for CSCW applications that require the use of multiple channels of information.

The move towards the Internet has given us the opportunity for global participation in CSCW environments, resulting in a more generic utilization of this technology. However, the caveat for the CSCW community lies in the fact that we now no longer fully control our shared environments, and have become more dependent on the global decision-making process regarding network infrastructure. The same institution that gave our research community access to internetworking in the late-1970s, the National Science Foundation (NSF), has decided that further governmental funding of the NSFNET backbone, which constituted a major part of Internet infrastructure in the USA, is no longer required. Commercial service providers now operate major backbone services for the Internet on a commercial basis [8]. These commercial network providers thus take charge over essential pieces of Internet infrastructure, taking decisions which could have a serious impact on distributed multimedia services provided by the Internet such as the World-Wide Web and MBONE, which we'll discuss further on. In Europe, a similar development is taking place: traditional, often monopoly-based, network providers such as British Telecom and the Dutch PTT Telecom have recently been privatised and are in the process of reconsidering their tasks and services. The debate in both the USA and Europe over the Information Super Highway or National Information Infrastructure (NII) gives further evidence that governments will not be able to maintain development and support of new high-bandwidth information services in the near future.

This, however, is only one network-related development that directly concerns the CSCW community. New standards are emerging for the transmission of real-time high-bandwidth data over Internet connections. This high-bandwidth data typically involve video and audio information from shared multimedia communication environments. In the next section, we will discuss what these standards might provide in terms of functionality to the CSCW community, and what constraints these standards introduce. They are often defined by network researchers who have a genuine interest in providing network capabilities, but typically do 
not have the same means as the CSCW community to regard cognitive ergonomical aspects of network functionality. We try to demonstrate the importance of a dialogue between the two areas of research, a cross-fertilization from which both communities will benefit.

The current attitude in the CSCW community towards connectivity is very similar to the attitude towards computing power in the field of Human-Computer Interaction ( $\mathrm{HCI})$ in the late 1970s: the infrastructure necessary to apply our research ideas is not of our concern, and will be delivered by others. That may have been true for direct manipulation systems, but to what extend will it be true for shared real-time multimedia environments?

\section{Networking Standards}

The world of international or global networking relies on the work of standard and regulatory bodies. These are the groups that set the recommendations that govern what the networks can offer. In most cases they are the representatives of governments or carrier organisations and their role is to ensure that the technicalities of the global network are compatible and professional. The International Telecommunication Union (ITU) [4], under the auspices of the United Nation, has created the ITU-T to look after the standardization sector of telecommunications. This was formally the role of the International Consultative Telegraph and Telephone Committee (CCITT). The ITU is made of members from the telecommunication suppliers and carriers, and is obviously a representative of these telecom consortiums. The ITU ensures proper functioning of the global telecommunication infrastructure, which is achieved by the harmonization of the various national policies. It does not normally carry the task beyond that of the 'basic' carrier service, as seen by the telecommunication companies. Because of this, the ITU normally deals with the Wide Area interconnections of the network infrastructure and studies the technical, operational and charging issues that arise from such services. From these studies it makes recommendations to its members in its harmonizing role. As a result of this, however, real end-user services are not normally addressed, as the ITU's endusers are the telecommunication companies. It must be stressed that the ITU only produces recommendations which are not binding. Yet these recommendations are generally complied with, since they guarantee interconnectivity of networks. This provides the foundation for enabling services to be provided on a worldwide scale.

The International Standards Organisation (ISO) also has a global task, in its role as an international standards body. As with the II'U, the ISO takes a professional line on ensuring the standards in global infrastructure. To assist the international aspects of this work, the ISO looks very carefully at other standard setring bodies around the world. A prime example of this is the adoption of the IEEE local area networking standards into the international community. The ISO has a much wider view of the world. It regards anyone as a potential user, and has therefore also included user services in their standards on networking. To that end the ISO does look at the end-user services which networks can implement across all networking infrastructures. Yet it appears that their recommendations often result in a set of user services which are not implemented due to the fact that they either duplicate, complicate or restrict services which are already in place. ISO recommenda- tions for new services often seem not acceptable either, or rely on other ISO defined services which have been slow to be taken up. For example, in order to establish the X400 electronic mail standard, the X500 standard for electronic directory services first needed to be implemented. However, work by the ISO has shown the need for certain services. For example, the work related to the problems of directory services (X500) has been taken up in other networking groups, such as SOLO and whoist+. Also, the ISO has the ability to swing governments. The acceptance of the Open Systems Interconnection (OSI) Reference model is a good example of this.

The world of the Internet has the Internet Engineering Task Force (IETF) and the elite Internet Research Task Force (IRTF) which are working to ensure that the tasks required to make the Internet work are investigated, discussed and undertaken. The means of the Requests for Comments (RFC) to elicit the end-users' views would appear to work well. The members of the Internet teams are extremely active, so progress is fast and developments occur at a rapid rate. Their motto, 'if it needs to be done, suggest how it might be done, try it, see if it works, and offer it to the community', results in a large selection of tools becoming available, but also results in a high mortality rate.

\section{An Example CSCW Network Standard: The MBONE}

Currently the Multicast Backbone (MBONE) $[1 ; 2 ; 9]$ is a very popular outcome of this work, and there is a major collection of tools that support the usage of the MBONE. The MBONE is a set of network connections which use a single address to fan out information which must reach its destinations within a given "lifetime". The destinations are all machines set up to accept the multicast transmissions on the Internet. Thus a single source of transmission can be fanned out through the Internet to millions of receiving host machines. This gives the ability to broadcast information very efficiently from one to many hosts. Here is a case in point, where the IETF community recognized a need for a service to create a situation where more Internet users could contribute to their own IETF meetings. Considering they were meeting about the Internet, it only seemed natural to utilize this resource to achieve their aim. Steve Casner established the workings of the service we now call the MBONE in 1992. Several tools have been developed to make use of the MBONE as a means of distributing both voice and video image, via software. Examples tools are Visual Audio Tool (VAT) [6] which allows sound to be transmitted over the Internet, Network Video (NV) [3] which allows video images to be transmitted over the Internet, the Session Directory (SD) [5] which allows the advertising of multicast sessions and the Whiteboard (WB) which allows interactive drawing across the Internet, to name just a few. Each tool looks after one particular medium, so that one medium does not impose restrictions upon another.

Although the IETF is quite aware of the problem of, for instance, multimedia synchronisation [10], its main focus is the impact of the media on the carrier network. For the moment, basic problems such as data loss and timeliness of data arrival continue to affect audio-visual communication over the Internet. The VAT audioconferencing program is usable in a point-to-point environment, but can be very disconcerting in a multiple user environment. However, video transmissions are the services that really show end- 
users what poor service the network will provide. Currently this service is based on partial updates of an image. This, combined with image compression, still results in approximately 1 to 4 frames a second which cannot be compared with broadcast quality output of approximately 25 full frames a second. The result is a 'blocky' image with apparent intermittent updates at random points. Put any workstation user in front of an ISDN video telephone and watch their response.

\section{Carrier Companies}

Telephone and data communication infrastructure is generally provided by carrier companies that own and operate the switching, maintenance, billing and transmission systems required to set up telephone and data connections. Example carriers are the Bell Operating Companies in the USA, British Telecom in the United Kingdom and PTT Telecom in the Netherlands. These companies have long been involved in the provision of network infrastructure to network providers on the basis of lease contracts. However, with the emerging need for new network infrastructure based on high-bandwidth, fibre-optic technology such as ATM, we signal a trend where carrier companies themselves are progressively becoming network providers. Depending on local circumstances, two main causes can be identified for this trend:

- The enormous investments involved in the construction of the new infrastructure;

- The integrated nature of high-bandwidth technology: the same network supports many types of traffic, including data, fax, CD-quality audio and real-time video information.

Non-profit organizations such as governments and universities only have the financial means to participate in the setup of highbandwidth network technology. In the United States, AT\&T owns much of the new high-bandwidth infrastructure. However, due to anti-trust regulations there (which caused AT\&T to be split up into seven smaller carrier companies), comperition in the States is growing, especially where the former NSFNET Internet backbone is concerned. In Europe, the privatisation of state-owned, monopoly-based carriers has been a relatively recent event. In The Netherlands, for example, the now privatised PTT Telecom will retain its monopoly on telephony-based applications until the end of this decade. This is why the Dutch ATM backbone, under development in the SURFnet 4 high-bandwidth infrastructure project, will be owned and operated by PTT Telecom. Incidentally, the same carrier company has a $49 \%$ interest in the (nonprofit) Dutch academic network operator SURFNET bv, which carries out the project [7].

So it seems, in The Netherlands at least, that carrier companies are going to play a critical role in deciding the future of telecommunications functionality. The CSCW community may wish that desktop computers become the focal point of telecommunication services, but do carrier companies share this view? How will they benefit from the use of distributed multimedia in CSCW applications, particularly long-distance video conferencing? In an attempt to answer the first question, we will discuss two examples of research at a typical European carrier company. As far as the second question is concerned, we can only speculate. We will discuss some possibilities, sketch a worst-case scenario and we will discuss what would remain of current-day CSCW functionality if this scenario is enacted.

\section{Carrier Company Research}

Although we are aware that the two projects discussed in this section by no means constitute a representative sample of CSCW research at carrier company laboratories, we feel the constraints put forward in these particular cases may provide us with an insight into some of their viewpoints on CSCW research and furure implementations.

\section{A Usability Test of a Phone-based ISDN Front-End}

In this recent project, the usability of ISDN supplementary services for telephony-based applications such as Call Forwarding (which allows the user to transfer incoming calls to another telephone automatically) was investigated by means of a well-conducted experimental study. The user interface was based on a computer-based (Hypercard) touch screen simulation of a telephone interface extended with a 40 digit display and voice instructions. Users could activate supplementary services by pressing number codes on the simulated keypad. Two factors were studied: the effects of presenting an explicitly structured conceptual model of the command language in the system's meta-communication, and the effect of age on the usability of the system. What is interesting about this study is that although it is concluded that this type of phone-based command language is very hard to use (particularly for elderly or less experienced users), no recommendations or comparisons were made with regard to alternative, nonphone-based (graphical) user interfaces. Instead, an improvement of the structuring of meta-information given by the phone-based system was considered an option to alleviate some of the inherent usability problems of this type of minimal interface.

\section{A Usability Test of a Desktop Conferencing System}

This project involved a usability testing of the Multipoint Interactive Multimedia Interpersonal System (MIMIS), part of the European RACE 2025 project. In this project, an integrated multimedia software environment is being developed for ATMnetworked workstations. The software allows users, amongst other things, to video-conference, send data to each other and jointly edit files containing multiple media. In the usability test, a comprehensive set of tasks was investigated by means of analysis of video data, written observations and questionnaires. We will outline some of the observations from one of the project reports.

The debate on the National Information Infrastructure asserts that future CSCW functionality should be accessible to a large and representative part of the population. The subjects in this particular usability test, however, were mostly middle-aged males, working in the higher ranks of the organisation. All tasks were strucrured and coordinated by observers, present with the subjects in the experiment rooms to give instructions, answer questions and, of course, create written observations. However, this setup prohibited the gathering of essential information on cooperation between subjects. Since time measurements regarding task completion were not automated, observers were unable to gather any quantitative data. The reliability of the questionnaires used was never established: it is unknown on which scales these were based, and a 
majority of questions was positively stated. From the report, however, it became clear that the user interface of the MIMIS system does not live up to current design standards. If we want carrier companies to seriously consider CSCW desktop systems as a widely used front-end to their public high-bandwidth networks, we should at least try to provide them with incentives in order to stimulate proper research in this direction.

\section{Charging Systems}

So with the introduction of new high-bandwidth integrated services networks, what will happen to recent $\mathrm{CSCW}$-related developments on the Internet such as MBONE video-conferencing, whiteboards and the World-Wide Web? Indeed, what will happen to the Internet itself? Is everyone going to get the kind of functionality the research community has already become used to? We feel that, apart from strategic decisions on standards for user frontends by carrier companies and service providers, this will very much depend on the type of charging system that will be devised for integrated services. Simply stated: it seems unlikely that carrier companies, particularly those with a legislated monopoly on telephony-based services, are going to allow us to continue using the Internet to bypass their phone, fax and video-conferencing infrastructure for very much longer. So what might happen if carrier companies decide to charge for networking services on a quantitative basis?

Let us consider the use of the current telephone charging system. If carrier companies or service providers will start measuring the time spent on a data connection, they should be able to determine whether a connection is real-time, or what data is being transferred. The inherent flexibility of distributed computer systems in the encoding of data makes it relatively easy for users to circumvent this type of restriction. Alternatively, they should be able to guarantee customers fixed transfer rates. Again, this seems highly unlikely with the inherent mesh topology of a global network. Transfer rates and latency depend, amongst other things, upon traffic and routing, and both will remain unpredictable. Because of the same reasons, it is also not straightforward to measure the distance over which data has been transferred. So the only measure that is quantifiable is the amount of data actually transferred. But how are customers going to be able to predict the amount of data they will be transferring during a World-Wide Web session? If carrier companies are going to choose the wrong charging system, Cyberspace will be a thing of the past. Let us suppose carrier companies will charge for the amount of data transferred and the disrance over which it is transferred. A World-Wide Web user in Europe views a home page in the United States. On this page, there is a link to a high-quality graphics file in Australia. The user however thinks the connection is with the United States, and expects the graphics file to be compressed. However, the charges end up being for the monthly subscription, a connection to Australia via the United States, and twice the amount of data than expected. As a research community, we should take such issues into careful consideration because of the impact they could have on functionality in CSCW environments. If we ignore these issues, the very principles on which our research is based are at risk.

\section{Conclusions}

Researchers in the fields of CSCW and HCI, familiar with the social and ergonomical aspects of computer supported cooperative work, should start a dialogue with network researchers and engineers in both an academic and corporate setting. The focus of this discussion should be on the specification of human factors requirements for global internetworking, taking into account the various structural technical limitations. The objective should be two-fold:

- To adapt current networking standards so that they address the cognitive, ergonomical and social ecological needs of users working together in a distributed environment, in order to provide a solid foundation for end-user service standards.

- To create a setting in which carrier service providers can implement this first objective on a global scale. If this setting is to be market-oriented, provisions should be taken that this market is a competitive one. This way, market forces might provide a fertile ground for real innovations in the way people will work together using computer systems, following the example of the emergence of direct manipulation systems over the past decade.

Of course such a cross-fertilization implies a trade-off for both sides. The CSCW community should take into account that network bandwidth might never be infinite and that there will always be latency in the transport of data. They should accept that all machines are not connected on a local network and that standards such as ISDN and H261 will also be used to interconnect machines. The ideal working environment of the academic CSCW practitioner may not be deliverable in the products that are required. This means that researchers in the field of CSCW should learn to deal with the structural technical limitations of networking technology as they are anticipated by networking people, and take these limitations into account when studying human factors in relation to such systems. On the other hand, we hope this paper has made clear that networking people have yet to develop a more thorough regard of the user on top of their model of the network. Although they should adhere to their focus on the provision of transport services, they should acknowledge the importance of human factors, and should realize that highly technical decisions can have a considerable impact on the end-users' ability to work with a system. To this extent, they should utilize the considerable human factors expertise already available in, for example, the academic environment, and not try to re-invent the wheel themselves.

\section{Acknowledgements}

We would like to thank Gerrit van der Veer and Bill Hefley for their helpful suggestions and support. We would also like to thank the members of the European Community COST-14 IMPACT group without whom this paper would never have been written.

\section{References}

[1] Deering, S. (1993). MBONE: The multicast backbone. Available as URL: ftp://parcftp.xerox.com/pub/net-research/cerfnetseminar-slides.ps.z 
[2] Ericksson, H. (1994). MBONE: The Multicast Backbone. Communications of ACM 37(8): 54-60.

[3] Frederick, R. (1994). nv: Networking video tool. Available as URL ftp://parcftp.xerox.com//pub/net-research/nv-paper.ps

[4] ITU (1994). General Information. Available as URL http:// info.itu.ch/

[5] Jacobson, V. (1993). sd: The session directory tool v1.13beta. Binaries for SPARC, DEC MIPS and Alpha, SGI MIPS and HP PA architectures available as URL: ftp://ftp.ee.lbl.gov/conferencing/sd/

[6] Jacobson, V. and McCanne, S. (1994). vat: Visual audio tool. Available from URL: ftp://ftp.ee.lbl.gov/conferencing/vat/

[7] Jurg, P. and Zegwaart, E. (1994). Innovatie van de SURFnet infrastructuur. Informatie: (Technische) infrastructuren 36(12): 833-842. (In Dutch)

[8] Kahn, R. E. (1994). Viewpoint: The Role of Government in the Evolution of the Internet. Communications of ACM 37(8): 15-19.

[9] Macedonia, M. R. and Brutzman, D. P. (1994). MBONE provides audio and video over the Internet. IEEE Computer. Available as URL: ftp://tauras.cs.navy.mil/pub/mbmg/mbone.html/: pp 30-36.

[10] Schulzinne, H., Casner, S., et al. (1995). RPT: A transport protocol for real-time applications. IETF draft. Available as URL: ftp:/ /ietf.cnri.reston.va.us/internet-drafts/draft-ietf-avt-rtp-07.toxt

\section{About the Authors}

Roel Vertegaal, M.Phil. (born 1968) is a research assistant and Ph.D. student with the Department of Ergonomics at the University of Twente, The Netherlands. After a degree in Music Technology at the Utrecht School of the Arts, The Netherlands, he studied
Computer Science at the University of Bradford, UK, where he researched the use of inpur devices in a multi-dimensional timbre (sound) manipulation task. His current project is on the use of mental representarions in the design of the system image (i.e., the perceived aspects of the user interface), with pilot projects relating to the representation of multidimensional sound spaces and the representation of distributed tasks such as video-conferencing.

Dr. Guest is a Computer Scientist who has been researching in Human-Computer Interaction (HCI) and Digital Communication for over 20 years. He was one of the Senior Research Fellows at the UK's only rolling grant funded centre for HCI. Since joining LUT he has been the grant holder of many UK and EU funded projects. These currently include JISC funded Resource Organisation and Discovery in Subject based Services (ROADS), the EU RACE II project Metaphors for Integrated Telecommunication Services (MITS) and EU COST 14 project Design of Computer Supported Cooperative Work.

\section{Authors' Addresses}

Roel Vertegaal

CTIT and Dept. of Ergonomics

University of Twente

P.O. Box 217

7500 AE Enschede, The Netherlands

Tel: +31-53 893324

Fax: +31-53356695

E-mail: R.Vertegaal@wmw.utwente.nl

Steve Guest

Dept. of Computer Studies

University of Technology

Loughborough, Leicestershire LE1 1 3TU

United Kingdom

Tel: +44-150922 2692

E-mail: S.P.Guest@lut.ac.uk 